\title{
Research of Water Quality Monitoring and Control System Based on STM32
}

\author{
Lei Zhang a, Xiaoning Chen \\ School of Anhui University, Anhui 230601, China. \\ a814043943@qq.com
}

Keywords: Sensor, water quality detection, microcontroller.

\begin{abstract}
With the advancement of science and technology, environmental pollution has also become more and more serious, especially the water resources closely related to human life are gradually being eroded by pollution. Monitoring the water quality of water resources becomes more urgent, but the traditional manual detection is not only time-consuming and laborious and cannot be real-time detection, the first time early warning, visual display of dynamic real-time curve diagram, and the error of the detection result is large, so according to actual needs and the current sensor for water quality testing has designed this water quality monitoring and control system. The system consists of various water quality detection sensors, SCM system, GPRS module, 485 communication module, solenoid valve group and other components. It can realize real-time collection and detection of water quality and water conditions in all weather conditions and transmit the collected data to the monitoring center for display, visual display, and early warning. High detection efficiency, low cost, worthy of promotion.
\end{abstract}

\section{Introduction}

The surrounding of the Foziling Reservoir in Huoshan County of Anhui Province is Dabieshan National Level Conservation Area, and the water quality of the reservoir is consistently superior to surface water standards, providing urban water for the downstream Hefei and Lu'an. The real-time detection and display of water quality parameters such as $\mathrm{pH}$, water temperature, conductance, dissolved oxygen, and chlorophyll are particularly important. The system can collect real-time information of water samples and carry out remote transmission under unsupervised supervision in the field, and finally display all water quality parameters in real time in the background.

\section{System Overall Structure}

The main control module uses ST's main chip based on ARM Cortex-M3 core, and the periphery is mainly composed of a relay group that controls the timed water collection, a GPRS module, and a 485-serial port. The sensor uses high-sensitivity, high-precision testing equipment produced by the company's Haxi company, which can accurately detect various water quality parameters in the water source. The main control chip collects water samples from the 10 meters, 6 meters, and 3 meters deep from the Foziling Reservoir through the relay timing control pump to the water sample detection tank. The water quality parameters of the water sample are detected by a high-precision sensor and detected. The water quality parameters are passed to the data acquisition instrument through the RS485 serial port, the data acquisition instrument is then passed through the RS485 interface microcontroller, and finally the single-chip microcomputer sends the collected data to the background terminal through the GPRS module. The terminal receives and processes the water sample information into the database. The monitoring department and users monitor the reservoir water quality dynamics in real time through monitoring and display software. The overall structure of the system is shown in Figure 1. 


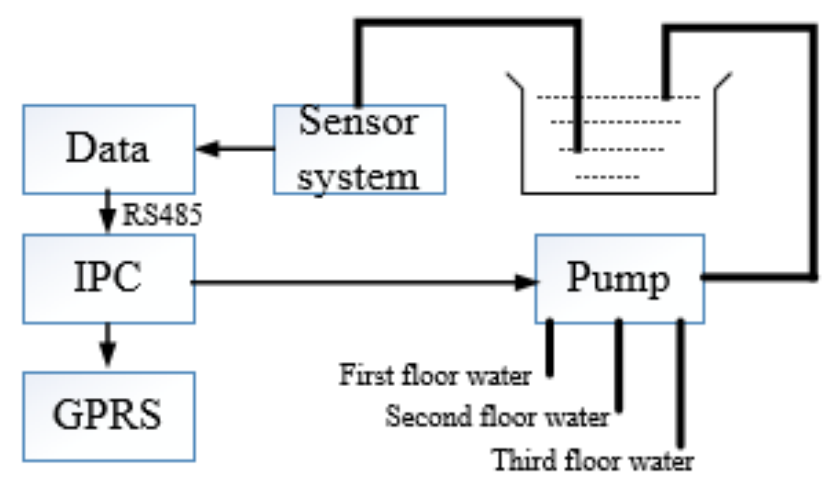

Fig 1. Overall structure of the system

\section{Hardware Circuit Design}

\subsection{Main Control Module.}

The STMicroelectronics STM32F103 family of chips is used, which is based on a 32-bit Flash microcontroller based on the ARM Cortex M processor core. The combination of high performance, real-time performance, digital signal processing, low power consumption, and low voltage while maintaining high integration and ease of development opens a whole new space for free development for MCU users and provides a variety of ease of use. Hardware and software aids. The main control module controls the on-off of the water pump indirectly by controlling the switch of the relay, to meet the requirements for sampling water samples at depths of 3 meters, 6 meters, and 10 meters at regular intervals. The MCU is connected to the sensor device through the RS485 interface. The device transmits the collected data to the SCM through the serial port 2. The SCM then transmits the received data through the serial port 1 to the background server through the GPRS module.

\subsection{Relay Control Module.}

Microcontroller is a weak electrical device, under normal circumstances most of the work in $5 \mathrm{~V}$ or even lower conditions. Drive current below mA level. However, it must be used to control the motor and some other high-power applications. Relay drive is a typical, simple power driver. The triode is equivalent to a switch, and the signal pin of the single-chip microcomputer outputs the high-low-level control triode to open and close, thereby controlling the relay to close to achieve the control effect. The relay control module is shown in Figure 2.

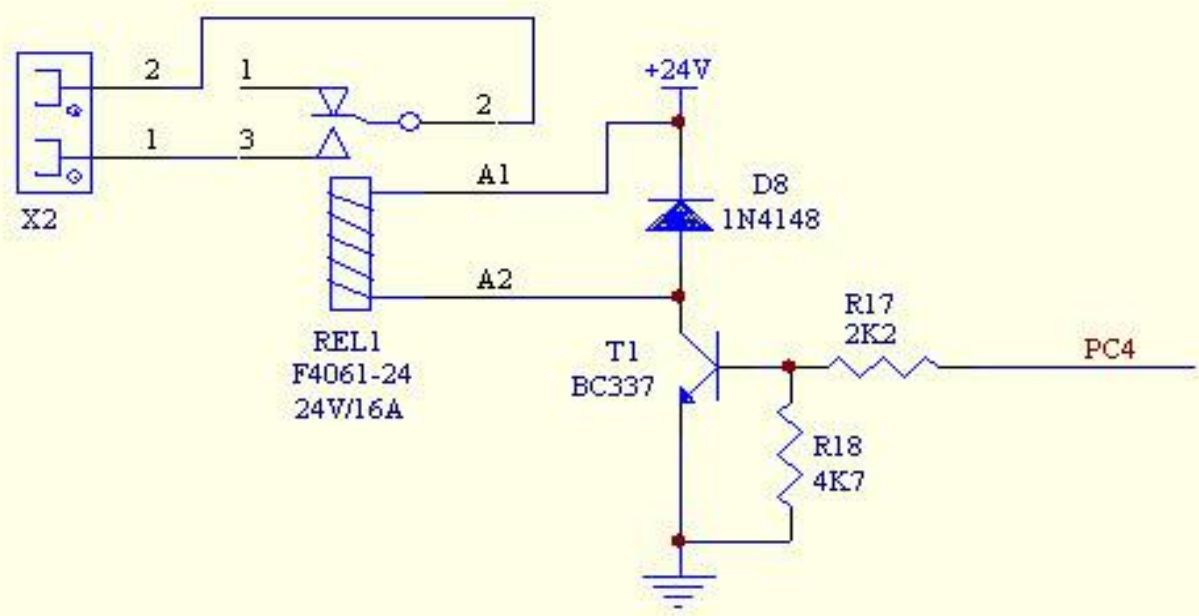

Fig 2. Relay control module

\subsection{GPRS Module.}

Data transmission adopts SIM900A module, SIM900A module is a compact GSM/GPRS module, adopts SMT encapsulation, STE-based single-chip solution, powerful performance, built-in customer applications. Can be widely used in car tracking, fleet management, wireless POS, handheld PDA, 
smart meter reading and power monitoring and many other directions. The SIM900A is a practical GPRS module that allows data transmission after simple configuration. In this project, the GPRS module is connected to the serial port 1 of the microcontroller, and the TX and RX pins of GPRS are respectively connected to the RX and TX of the microcontroller. In the MCU, the AT command connected with GPRS is configured in advance, and 0x1a is transmitted for the last time to start data transmission. When the MCU receives the normal instruction returned by the SIM900A module to indicate that the connection is successful, the MCU can start transmitting the data collected by the sensor to the background through the GPRS module.

\subsection{RS485 Serial Communication Module.}

RS485 is a typical serial communication standard. They define voltage, impedance, etc. but do not define the software protocol. It has the advantages of low interface level, non-destructive chip, high transmission rate, strong anti-interference ability, long transmission distance, and many support nodes. RS485 belongs to the one-chip computer peripheral circuit in this project, and connect with the RX, TX pin of the data acquisition instrument. The data acquisition instrument sends the water sample information collected from the water quality detection sensor to the single-chip microcomputer through the serial port and is processed by the single-chip microcomputer and then transmitted to the server through the GPRS module. The RS485 receives and sends data through a SP3485 transceiver that complies with the serial communication protocol. The SP3485 transceiver is shown in Figure 3 below. The A and B bus interfaces in the figure are used to connect the 485 buses. $\mathrm{RO}$ is the receiving output terminal, DI is the sending data receiving terminal, RE is the receiving enable signal (active low), and DE is the sending enable signal (active high).

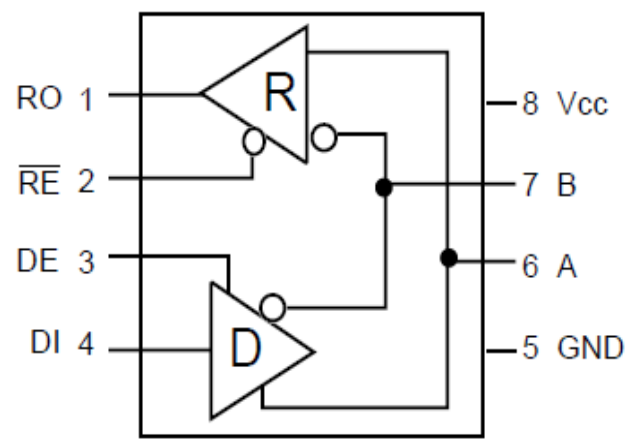

Fig 3. SP3485 transceiver

\section{Monitoring Center Station Overall Design}

The water quality online monitoring and management platform display interface is shown in Figure 4 below. The data transmitted by GPRS is received in the background and stored in the server, using SQL Server as the database management system. The water quality monitoring and management platform displays the received monitoring data in real time and the monitoring department and users can query the monitoring data through the platform. The monitoring platform mainly consists of four parts:

1). site monitoring:

Mainly divided into list monitoring, chart monitoring, real-time monitoring. Each water quality parameter can be visually displayed, and one type of monitoring data can also be selected and displayed in the form of a graph or a curve. When a certain monitoring data exceeds the limit, special marks can be displayed to facilitate the monitoring management personnel to grasp the real-time data.

2). data summary

Including historical data summary, historical data exceeded. The historical data of water quality are summarized, and the historical changes of water quality are graphically displayed in the form of a graph.

3). data analysis 
Automatically compares the collected data with historical data and analyzes changes in water quality parameters.

4). data reports

Display the historical data of the specified monitoring parameters, and automatically generate the historical data report of day, week, month and year.

\section{Conclusion}

The system uses a single-chip microcomputer as the main control chip and controls the water pump to carry out stratified pumping of water samples through weak electric power control. The water quality parameters of different layers were detected by the sensor equipment and the data obtained was transmitted to the single-chip microcomputer through RS485. The SCM is sent to the GPRS module through the serial port and sent by the GPRS module to the backend server. Realization of automatic control and real-time data acquisition, which avoids the low manual efficiency and inaccuracy of detection, is worth promoting.

\section{References}

[1]. Fei Shao, Jinliang Wang, Bin Mei, Bingjie Wang. Water Pollution Remote Monitoring System Based on STM32. Private technology. Vol. 11 (2016) No. 7, p. 73-74.

[2]. Jianbo Shao, Xiaofei Lu, Jiantao Shao. Design of Multi-parameter Water Quality Monitoring System Based on STM32. Heilongjiang Science and Technology Information. Vol. 21 (2011) No. 12, p. 48-50.

[3]. Qing Li: Research and Design of Intelligent Water Quality Monitoring System Based on STM32 (Master of Engineering, Hefei University of Technology, China 2015. p.15-45. 